\title{
QUOTIENT GROUPS OF TOPOLOGICAL GROUPS WITH NO SMALL SUBGROUPS
}

\author{
SIDNEY A. MORRIS
}

Abstract. It is shown here that a quotient group of a topological group with no small subgroups can have small subgroups.

It is well known that a quotient group of a Lie group is a Lie group, or equivalently ([2], [6]) that a quotient group of a locally compact group with no small subgroups is a locally compact group with no small subgroups. Irving Kaplansky [3] asks: if $G$ is a topological group with no small subgroups and $H$ is a closed normal subgroup of $G$, is $G / H$ (necessarily) a group with no small subgroups? We answer the question in the negative here.

THEOREM. If $X$ is any metric space and $F(X)$ is the free abelian topological group on $X[4]$, then $F(X)$ has no small subgroups.

Proof. By the Arens-Eells embedding theorem ([5], [1]) $X$ can be embedded isometrically in a normed linear space $N$ as a Hamel basis. Let $S$ be the subgroup of (the additive group structure of) $N$ generated by $X$. We claim that $N$, and hence $S$, has no small subgroups. This is seen by noting that the unit ball of $N$ contains no nontrivial subgroups.

Since $F(X)$ is the free abelian topological group on $X$, there exists a continuous homomorphism $f$ of $F(X)$ onto $S$ which acts identically on $X$. Using the fact that $X$ is a Hamel basis for $N$, we see that $f$ is an algebraic isomorphism. Consequently $F(X)$ has no small subgroups.

EXAMPLE. Let $X$ be the cartesian product of a countably-infinite family of topological groups, each of which is topologically isomorphic to the circle group with its usual topology. Clearly $X$ is a topological group with small subgroups.

Since $X$ is a metric space, by the above theorem, $F(X)$ has no small subgroups. Finally we note that, by Theorem 23 of [4], $X$ is a quotient group of $F(X)$.

Received by the editors April 19, 1971.

AMS 1970 subject classifications. Primary 22A05, 22E15.

Key words and phrases. Topological group with no small subgroups, free abelian topological group, quotient group.

(c) American Mathematical Society 1972 


\section{REFERENCES}

1. R. F. Arens and J. Eells, Jr., On embedding uniform and topological spaces, Pacific J. Math. 6 (1956), 397-403. MR 18, 406.

2. A. Gleason, Groups without small subgroups, Ann. of Math. (2) 56 (1952), 193-212. MR 14, 135.

3. Irving Kaplansky, Lie algebras and locally compact groups, Univ. of Chicago Press, Chicago, 1971.

4. A. A. Markov, On free topological groups, Izv. Akad. Nauk SSSR Ser. Mat. 9 (1945), 3-64; English transl., Amer. Math. Soc. Transl. (1) 8 (1962), 195-272. MR 7, 7.

5. E. Michael, $A$ short proof of the Arens-Eells embedding theorem, Proc. Amer. Math. Soc. 15 (1964), 415-416. MR 28 \#5421.

6. D. Montgomery and L. Zippin, Small subgroups of finite-dimensional groups, Ann. of Math. (2) 56 (1952), 213-241. MR 14, 135.

Department of Mathematics, University of Florida, Gainesville, Florida 32601

Current address: Department of Mathematics, University of New South Wales, Kensington, N.S.W., 2033 Australia 